Document downloaded from:

http://hdl.handle.net/10251/108478

This paper must be cited as:

Prats-Boluda, G.; Ye Lin, Y.; Bueno Barrachina, JM.; Rodríguez De Sanabria Gil, R.; GarciaCasado, J. (2016). Towards the clinical use of concentric electrodes in ECG recordings: influence of ring dimensions and electrode position. Measurement Science and Technology. 27(2):1-11. doi:10.1088/0957-0233/27/2/025705

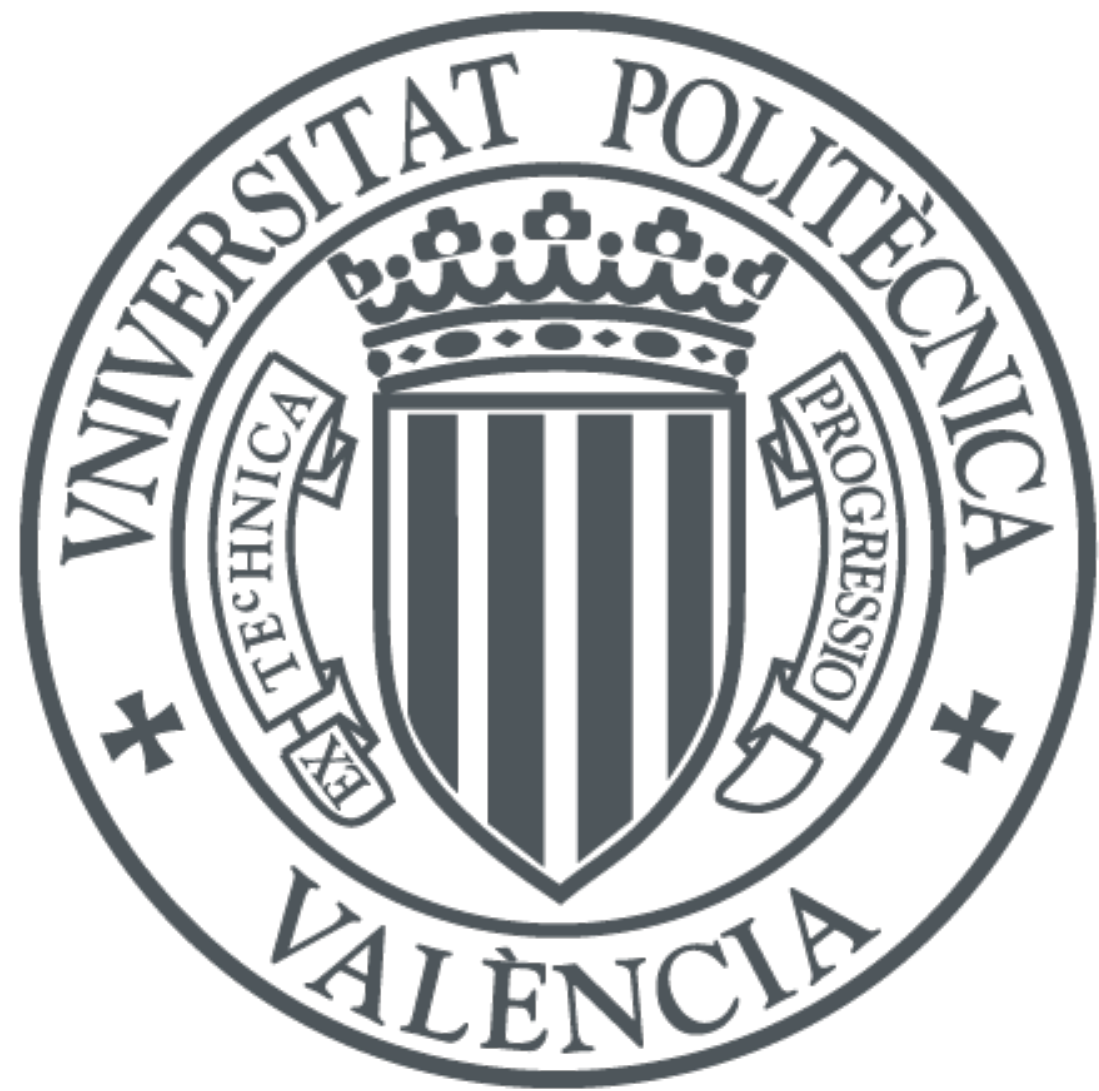

The final publication is available at

http://doi.org/10.1088/0957-0233/27/2/025705

Copyright IOP Publishing

Additional Information 


\title{
Towards the clinical use of concentric electrodes in ECG recordings: Influence of ring dimensions and electrode position
}

\author{
G Prats-Boluda ${ }^{1}$, Y Ye-Lin ${ }^{1}$, JM Bueno-Barrachina ${ }^{1}$, R Rodriguez de Sanabria ${ }^{1}$ and $\mathbf{J}$ \\ Garcia-Casado ${ }^{1}$ \\ ${ }^{1}$ Centro de Investigación e Innovación en Bioingeniería. Universidad Politécnica de Valencia, \\ Camino de Vera s/n Ed.8B, 46022 Valencia, Spain \\ Email: geprabo@eln.upv.es
}

\begin{abstract}
To overcome the limited spatial resolution of standard 12-lead ECG recordings, concentric ring electrodes (CRE) have been proposed to provide valuable data for the diagnosis of a wide range of cardiac abnormalities, including infarction and arrhythmia. Although theoretical studies indicate that the dimensions of the CRE regulate the depth of the electric dipoles sensed by these electrodes, this has not been experimentally confirmed. The aim of this work was to analyze the influence of CRE dimensions and position of a wireless multi-CRE sensor node on the cardiac signal recorded. For this, four wireless multichannel ECG recording nodes based on flexible multi-ring electrodes were placed at positions CMV1 (position comparable to V1), CMV2, CMV4R and CMV5; each node providing three bipolar concentric ECG signals (BC-ECG). Standard 12-lead ECG and 12 BC-ECG signals were recorded in 29 volunteers. The results revealed that a ring with an outer diameter of $33.5 \mathrm{~mm}$ achieves a balance between the ease-of-use and spatial resolution of smaller electrodes and improved detectability and higher amplitudes of signals from larger ring electrodes. Although a standard 12-lead ECG outperforms BC-ECC recordings in detectability of cardiac waves, if the relative amplitude of the wave is also considered, BC-ECG at CMV1 proved superior at picking up atrial activity. In fact, in most of the BC-ECG signals picked up at CMV1, P1 and P2 atrial activity waves were more clearly identified than in simultaneous 12-Lead ECG signals. Likewise, BC-ECG signals revealed higher spatial resolution in detecting anomalous electrical activity in local regions, such as impaired intraventricular driving, or atrioventricular blocks. Finally, the wireless multiCRE sensor node provides enhanced comfort and handling to both patient and clinician over wired systems.
\end{abstract}

Keywords: Multi-CRE electrode; BC-ECG recording, local cardiac activity.

\section{Introduction}

Cardiovascular disease remains the major cause of disability and premature death throughout the world. Coronary heart diseases are typically diagnosed after a risk assessment. The standard non-invasive 12-Lead ECG is currently the most widely used method of diagnosing cardiac disorders, despite the fact that the literature has shown that it provides limited spatial information for detecting local ventricular, and even less atrial, cardiac activity [1-2]. This is mainly due to the use of disk electrodes and their poor spatial selectivity in picking up electric dipoles because of the blurring effect associated with the different conductivities of the volume conductor [3-6]. 
The clinical diagnosis of pathologies associated with regions of anomalous local electrical activity, such as ventricular arrhythmias, ventricular ischemias, fibrillation, atrial flutters and atrial hypertrophies, require invasive electrophysiology which involves significant risks for the patient and increases the time of diagnosis [7]. Therefore high spatial resolution non-invasive monitoring systems would be very useful in the diagnosis of these pathologies. In this regard, the literature has proven that body surface potential maps (BSPM), obtained by recording signals from tens of electrode locations on the torso, provide diagnostic information not present in 12-lead standard systems that could be useful in the diagnosis of the above-mentioned pathologies and disorders [8-10]. Nonetheless, the spatial resolution obtained by merely increasing the number of recording electrodes on the body surface is limited, due to the smearing effect caused by the torso volume conductor [2]. This limitation can be overcome by obtaining body surface Laplacian potential recordings. The Laplacian of surface potential is the second spatial derivative of the potentials on the body surface and can be interpreted as a filter that allocates more weight to the bioelectrical dipoles adjacent to the recording points and provides more detail in differentiating multiple concurrent dipole sources [3]. Body surface Laplacian maps (BSLM) were drawn by using large numbers of monopolar electrodes on the chest and applying discrete approximations [3,5,11]. Nevertheless, the extensive use of BSPM or BSLM as diagnostic tools has been hindered by the inconvenience of applying such a large number of electrodes to the surface of the torso in clinical practice, which is time-consuming and annoying for both clinician and patient. In this context, active sensors based on concentric ring electrodes (CRE) in bipolar and tripolar configurations were proposed to estimate the Laplacian potential and acquire more localized electrical activity than conventional disk electrodes, alleviating orientation problems and reducing the amount of mutual information required by disk electrodes $[4,6,12,13]$.

However, certain aspects seem to complicate the use of CRE electrodes in clinical practice. First of all, although computational studies have proven that the size of the CRE is closely related to the electric dipole depth sensed by these electrodes [14], no experimental results have been reported to confirm this. In this respect, the use of multi-CREs would allow an assessment of the influence of electrode size on the bioelectrical signal sensed by a CRE. Secondly notwithstanding their enhanced spatial selectivity, the amplitude of the bioelectrical signal recorded by a CRE is smaller than those recorded with conventional electrodes of the same size [15]. As a result, high precision instrumentation and transmission systems are required to reduce electronic noise and interference in CRE recordings. Descriptions can be found in the literature of active sensors made of a reusable preamplifier circuit placed on the back of the CRE and wired to a benchtop bioamplifiers for additional analog processing [2]. However, more comfortable and easy-to-use systems are required in a clinical environment, so that sensor node prototypes made of tripolar ring electrodes directly connected to an acquisition and conditioning module that transmits the captured data wirelessly have been developed and tested in ECG recordings [16]. Conditioning modules with better performance (higher number of input channels and bandwidth) are required for use with multi-CREs. Finally, no comparative studies that analyzed the influence of CRE size and location on the bipolar concentric electrocardiographic (BC-ECG) signals have been found in the literature regarding the detection capabilities and features of ECG recordings from CRE and standard 12-Lead ECG.

Therefore, the aim of this work was to analyze the influence of CRE dimensions and the position of a wireless multi-CRE sensor node on the BC-ECG cardiac signals recorded. These signals were also compared with standard 12-Lead ECG recordings. 
Three BC-ECG signals are obtained from the multi-CRE sensor node:

$$
\begin{aligned}
& \mathrm{BC} 1=\mathrm{U}_{2}-\mathrm{U}_{1} \\
& \mathrm{BC} 2=\mathrm{U}_{3}-\mathrm{U}_{1} \\
& \text { BC3 }=\mathrm{U}_{4}-\mathrm{U}_{1}
\end{aligned}
$$

Where $U_{1}, U_{2}, U_{3}$ and $U_{4}$ are the biopotentials picked up by the inner disk and the three middle hooks (from the inside out) of the multi-CRE, respectively. Using the three-electrode 
technique for biosignal acquisition, the outer hook, $\mathrm{U}_{5}$, was connected to the circuit analog ground to reduce common mode noises, so that no additional external reference electrode was required. The custom-made compact signal conditioning and recording system wirelessly (Bluetooth) transmits online signals and stores them locally in a microSD card, thus avoiding the inconvenience of benchtop equipment. The circuitry includes bandpass filtering between 0.3 and $150 \mathrm{~Hz}$ and a gain of $4084 \mathrm{~V} / \mathrm{V}$. The sensed bioelectric signals are digitalized at $500 \mathrm{~Hz}$ with 24-bit resolution. The system is powered by a $3.7 \mathrm{~V}$ rechargeable $1000 \mathrm{mAh}$ Lithium Polymer battery, which ensures patients' safety.

\subsection{ECG signal recording}

Twenty nine recording sessions were carried out on 23 male and 6 female volunteer subjects in the Juana Portaceli Health Center of the Polytechnic University of Valencia (aged between 24 and 70 years old and with BMIs between 19.07 and $33 \mathrm{Kg} / \mathrm{m}^{2}$ ), who had attended routine check-ups, some healthy and others with cardiac pathologies. This study was approved by the Universidad Politécnica Ethics Committee and adhered to the Declaration of Helsinki. The volunteers were informed of the nature of the study, briefed on the recording protocol and signed a consent form. The recordings were conducted with the subjects lying on a stretcher.

For each subject, standard 12-Lead electrocardiographic signals (ECG 8270 monitor, Nihon Kohden, Japan) and 12 BC-ECG recordings from four dry wireless multi-CRE sensor nodes, placed in positions shown in Figure 2, were recorded, conditioned and wirelessly transmitted. BC-ECG signals were identified according to the hook used for obtaining the potential difference (BC1, BC2 and BC3) and on the location of the electrode, as follows: - for CMV1 (position comparable to V1 near to the atria -right): BC1.1, BC2.1, BC3.1, - for CMV2 (comparable to V2, near to atria -left): BC1.2, BC2.2, BC3.2), - for CMV4R (comparable to V4R, exploratory placement): BC1.4, BC2.4, BC3.4, - for CMV5 (comparable to V5, near to ventricles): BC1.5, BC2.5, BC3.5). The skin area on which the electrodes (conventional and concentric) were placed was previously minimally exfoliated (Nuprep, Weaver and Company, USA) and in the case of male volunteers was also shaved to reduce contact impedance. The subjects' skin was then thoroughly cleaned with alcohol. The multi-CREs were attached to the chest with surgical tape, without applying any additional pressure. Immediately after placing the electrodes the 5-min recording session began. The duration of the whole recording protocol (skin preparation, positioning and recording) was about 15 minutes. 


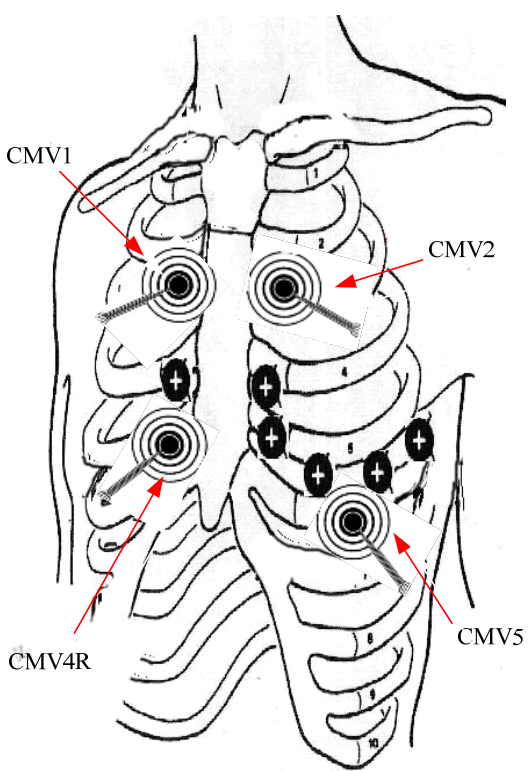

Figure 2. Diagram with the arrangement of the monopolar electrodes in standard precordial positions (V1, V2, V3, V4, V5 and V6) and the four wireless multi-CRE sensor nodes in positions CMV1 (comparable to V1), CMV2 (comparable to V2), CMV4R (comparable to V4R) and CMV5 (comparable to V5) for electrocardiographic signals recording.

\subsection{Data analysis}

In order to compare the applicability of the BC-ECG signals with standard electrocardiographic recordings, the following parameters were defined.

- Detectability (D) of the P, Q, R, S and T waves of the cardiac heartbeat in the 12-Lead standard recordings and in BC-ECG signals [17-19]. 12-lead standard ECG signals were recorded using an electrocardiograph device commonly available in health care centers, whose output is usually acquired on paper and not in digital format. In this work, the identification of the PQRST waves (detectability) in both standard and BC-ECG recordings was performed by an experienced cardiologist, who determined whether or not each wave was distinguishable in every heartbeat using the paper recordings of the signals.

- Median peak-to-peak amplitude for each ECG beat of the 12 standard ECG and the 12 BCECG signals.

- Normalized amplitude (NA) of each detected wave with respect to the peak to peak amplitude (RS). A common procedure used in cardiology to identify and analyze PQRST waves when evaluating electrocardiographic recordings, is to adjust the vertical printing scale according to the heart signal's peak-to-peak amplitude in order to obtain a maximum visual span of the signal to allow the cardiologist to detect all the electrocardiographic waves. It is therefore important to quantify the normalized amplitude (NA) of each detected wave with respect to the maximum signal excursion, since it is directly related to their capacity for analysis. In other words, the higher the normalized amplitude of a wave, the easier it is to identify and assess its morphology, which would help in the diagnosis of cardiac pathologies. In this context, it should be remembered that the parameters related to normalized amplitude (or amplitude ratios) of the cardiac waves are widely used in the literature for different aims: ranging from the development of new diagnostic algorithms to biometric recognition systems [20,21]. 
- DxNA (Product of Detectability by Normalized Amplitude): An optimum ECG recording system would provide the highest values of both detectability (D) and normalized amplitude (NA) for each electrocardiographic wave. Hence the importance of finding a formula that combines both parameters, such as the product of DxNA.

\section{Results}

Figure 3 shows 2.5 seconds of 12 BC-ECG simultaneous signals captured with the 4 wireless multi-CRE sensor nodes placed at positions CMV1, CMV2, CMV4R and CMV5. First of all, it can be seen that the amplitude of the BC-ECG signals is smaller (between tens and hundreds of $\mu \mathrm{V}$ ) than those picked up by the conventional electrodes (units of $\mathrm{mV}$ ). This is mainly because of the fact that the distance between the different rings and the inner disk is much smaller than that between conventional monopolar electrodes and the reference electrode. Likewise, it can also be observed that the bigger the size of the outer hook, the higher the signal amplitude of the BC-ECG. Another issue to consider is that not all the electrocardiographic waves (P,Q,R,S and T) are identified in all the recording channels. For instance, in Figure 3, even P1 and P2 waves, showing the electrical activity of the right and left atria, can be clearly distinguished in BC2-ECG and BC3-ECG signals at CMV1 (BC2.1, BC3.1), but atrial activity cannot be identified by visual inspection in any of the BC-ECG signal recordings at the CMV5 position, due to the local bipolar resolution of the CRE. Furthermore, the BC-ECG signal morphology is seen to vary not only with the position of the multi-CRE sensor node, but in some cases there were wide variations in the morphology of signals picked up at the same position with the multi-CRE electrode, as shown in the CMV1 in Figure 3.

Tables 1 and 2 depict the detectability percentage of the electrocardiographic waves (P,Q,R,S and T) in the 12 BC-ECG recording channels and the 12-Lead standard ECG and for the total of the recording sessions. It can be observed that higher detectability percentages are achieved by the 12-Lead standard ECG system than the 12 BC-ECG signals. Indeed, the precordial leads V1, V2 and V3 show the highest detectability values for all waves except the S, whose detectability was highest in V5. As for the BC-ECG recordings, the signals picked up at the CMV1 and CMV2 locations present higher detectability percentages than those obtained at CMV4R and CMV5. Furthermore, for each multi-CRE, the bigger the size of the ring electrode and its distance from the central disk, the higher the detectability rate. In this regard the detectability values associated with BC1-ECG recordings are much lower than those achieved for BC2-ECG and BC3-ECG for all electrocardiographic waves (see Table 1).

As for ECG beat peak-to-peak amplitudes, in the case of the standard 12-lead recordings the highest values (18.5-17.8 mV) are those of the V3, V4 and V5 precordial leads, near the ventricles, and the lowest value is that of aVL $(6.5 \mathrm{mV})$. For BC-ECG recordings, the peak-to-peak amplitude is much lower (hundreds or tens of $\mu \mathrm{V}$ ), with CMV2 and CMV5 being the electrode locations with the highest amplitudes. It seems that the electrode at CMV2 is able to pick up ventricular activity even better than at CMV5. This may be because the relative distance between the multi-CRE and ventricles at CMV2 is smaller than at CMV5, due to the different anatomical characteristics of the subjects. As previously mentioned, Figure 4 also clearly shows that the larger the outer hook, the higher the BC-ECG signal amplitude, regardless of its position (CMV1, CMV2, CMV4R, or CMV5). 
CMV1

$\mathrm{BC} 1$

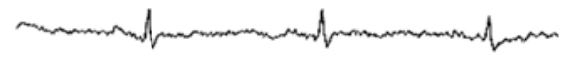

$\mathrm{BC} 2$

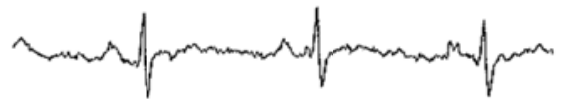

$\mathrm{BC} 3$

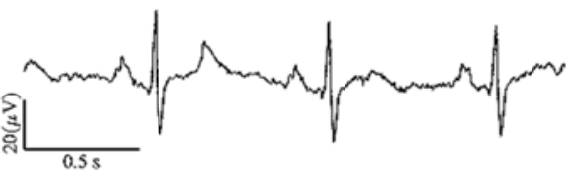

$\mathrm{BC} 1$

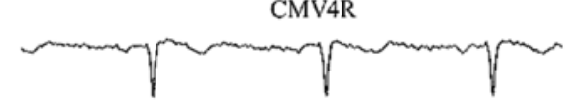

$\mathrm{BC} 2$

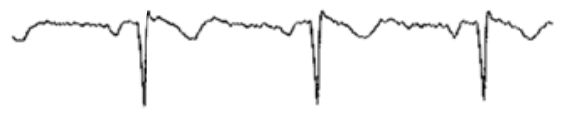

$\mathrm{BC} 3$

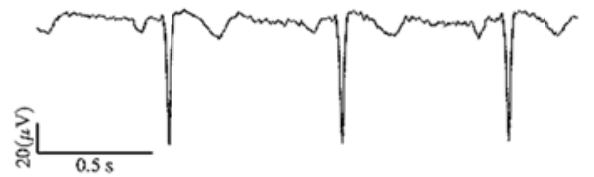

CMV2
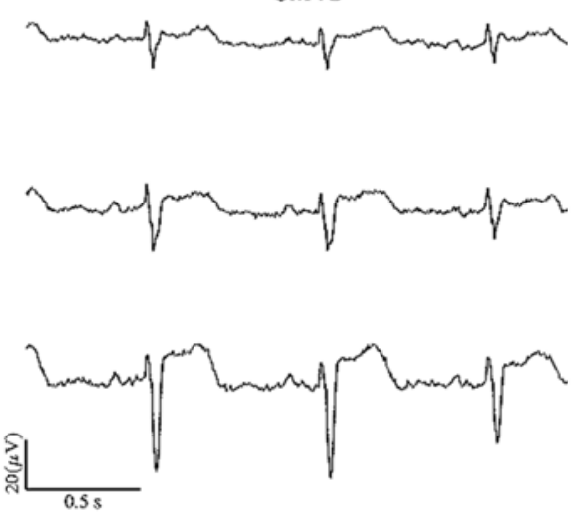

CMVs
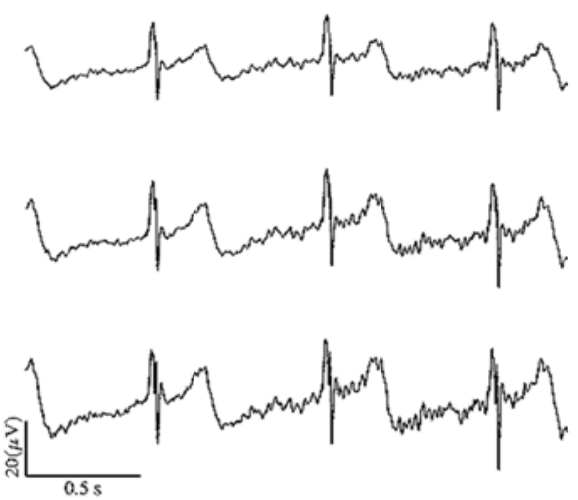

Figure 3. 2.5 seconds of 12 BC-ECG signals simultaneously captured with 4 wireless modules and multiCREs placed at positions CMV1, CMV2, CMV4R and CMV5. Patient had mitral valve prolapse.

Table 1. Detectability percentage of the electrocardiographic waves (P,Q,R,S and T) in the 12 BC-ECG recording channels.

\begin{tabular}{|c|c|c|c|c|c|c|c|c|c|c|c|c|c|c|}
\hline & \multicolumn{3}{|c|}{ CMV1 } & \multicolumn{3}{|c|}{ CMV2 } & \multicolumn{3}{|c|}{ CMV4R } & \multicolumn{3}{|c|}{ CMV5 } & \multirow[b]{2}{*}{$\begin{array}{l}\text { Max } \\
(\%)\end{array}$} & \multirow[b]{2}{*}{\begin{tabular}{|c|} 
Median \\
$(\%)$
\end{tabular}} \\
\hline $\begin{array}{ll}\text { Lead } \\
\text { Wave }\end{array}$ & BC3 & BC2 & $\mathrm{BC} 1$ & BC3 & BC2 & BC1 & BC3 & $\mathrm{BC} 2$ & BC1 & BC3 & BC2 & BC1 & & \\
\hline$P$ & 75,9 & 58,6 & 24,1 & 82,8 & 79,3 & 51,7 & 41,4 & 41,4 & 20,7 & 10,3 & 6,9 & $\beta, 5$ & 82,8 & 41,4 \\
\hline $\mathrm{Q}$ & 86,2 & 89,7 & 51,7 & 79,3 & 79,3 & 69,0 & 82,8 & 82,8 & 72,4 & 69,0 & 58,6 & 48,3 & 89,7 & 75,9 \\
\hline $\mathrm{R}$ & 96,6 & 100,0 & 69,0 & 100,0 & 100,0 & 100,0 & 96,6 & 96,6 & 89,7 & 100,0 & 100,0 & 96,5 & 100,0 & 98,3 \\
\hline $\mathrm{s}$ & 51,7 & 58,6 & 27,6 & 62,1 & 69,0 & 62,1 & 37,9 & 48,3 & 31,0 & 62,1 & 69,0 & 48,3 & 69,0 & 55,2 \\
\hline $\mathrm{T}$ & 75,9 & 69,0 & 31,0 & 89,7 & 89 & 75,9 & 86,2 & 75,9 & 55,2 & 82,8 & 69,0 & 55,2 & 89,7 & 75,9 \\
\hline
\end{tabular}

Table 2. Detectability percentage of the electrocardiographic waves (P,Q,R,S and T) in the 12-Lead standard ECG recording.

\begin{tabular}{|c|c|c|c|c|c|c|c|c|c|c|c|c|c|c|}
\hline $\begin{array}{l}\text { Lead } \\
\text { Wave }\end{array}$ & $\mathrm{V} 1$ & V2 & V3 & V4 & V5 & V6 & I & II & III & $\mathrm{aVR}$ & $\mathrm{aVL}$ & $\mathrm{aVF}$ & $\begin{array}{l}\text { Max } \\
(\%)\end{array}$ & $\begin{array}{c}\text { Median } \\
(\%)\end{array}$ \\
\hline$P$ & 86,2 & 79,3 & 82,7 & 86,2 & 79,3 & 75,7 & 79,3 & 96,5 & 65,5 & 96,5 & 55,2 & 82,8 & 96,5 & 81 \\
\hline Q & 100 & 93,1 & 89,6 & 6,9 & 27,6 & 51,7 & 55,2 & 51,3 & 62,1 & 51,7 & 69 & 55,2 & 100 & 55,2 \\
\hline $\mathrm{R}$ & 100 & 100 & 100 & 100 & 100 & 100 & 100 & 100 & 100 & 100 & 100 & 100 & 100 & 100 \\
\hline $\mathrm{s}$ & 20,6 & 24,1 & 10,3 & 82,7 & 86,2 & 68,9 & 48,3 & 58,6 & 51,7 & 58,6 & 44,8 & 51,2 & 86,2 & 51,45 \\
\hline $\mathrm{T}$ & 82,7 & 93,1 & 96,5 & 100 & 100 & 100 & 86,2 & 93,1 & 65,5 & 86,2 & 79,3 & 89,7 & 100 & 91,4 \\
\hline
\end{tabular}




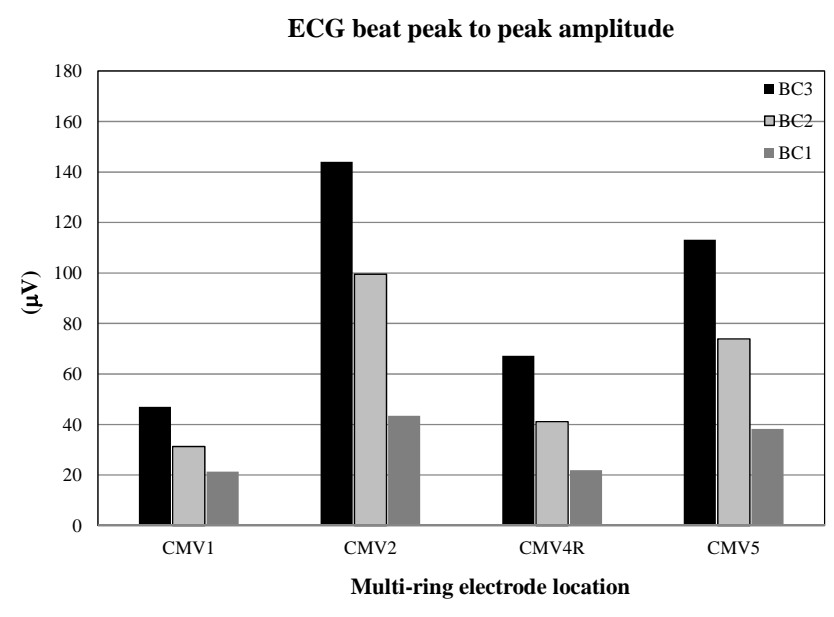

Figure 4. Bar chart of the median values of ECG beat peak-to-peak amplitude for 12 BC-ECG recordings in 29 subjects.

Figures 5 and 6 show the bar diagrams of the median values of the normalized amplitudes of the $\mathrm{P}, \mathrm{Q}, \mathrm{S}$ and $\mathrm{T}$ electrocardiographic waves for all recording channels (12 standard and 12 BC-ECG). It can be appreciated that the normalized amplitudes for the P wave of standard 12-Lead recordings are much lower than those of the $\mathrm{Q}, \mathrm{S}$, and $\mathrm{T}$ waves. Specifically, the maximum NA value for the P wave is 0.14 in Lead III, followed by 0.13 in Lead I and aVL 0.12, being 0.1 in aVR and aVF. In the case of precordial leads, the maximum value (0.09) is that of V1. The maximum NA of Q wave (0.51) is recorded in the V3 precordial lead, followed by 0.3 and 0.2 (Leads V2 and III). Maximum NA values for S and T waves $(0.49,0.52)$ are also obtained from V3 signals. NA values over 0.2 are reached in V1, V4, I, III, $\mathrm{aVL}$ and $\mathrm{aVF}$ for the $\mathrm{S}$ wave, whereas for the $\mathrm{T}$ wave NA values are higher than 0.2 in all leads with the exceptions of V1 and III.

In the case of BC-ECG signals, the highest normalized amplitudes for the $\mathrm{P}$ wave were obtained in CMV1. This result agrees with the fact that the maximum normalized value for the $\mathrm{P}$ wave in standard precordial leads is that of V1. It is remarkable that the NA values for the $\mathrm{P}$ wave in BC-ECG signals at CMV1 $(0.38,0.24,0.23)$ almost tripled those reached in standard 12-Lead recordings. Regarding the $\mathrm{Q}$ wave, as in the case of the $\mathrm{P}$ wave, it is best recorded in CMV1, with similar NA (0.53) to those achieved with standard leads (V3). It is possible that putting a multi-CRE at the V3 position would have given higher NA values for the $\mathrm{Q}$ wave. As for the $\mathrm{S}$ wave, the maximum NA was also at CMV1 (0.39), and was very similar to those obtained at CMV2 (0.35). It is noteworthy that in almost every recording position, the NA of the $\mathrm{S}$ wave exceeds 0.2. Comparing BC-ECG and 12-lead results, it can be deduced that higher values could have been reached by placing multi-CRE at CMV4 or CMV3. Finally, the T wave was recorded with the greatest normalized amplitude (0.49) at CMV2, similar to that achieved with the best standard lead ( 0.53 by V3), followed by CMV1 (0.4) with values over 0.2 in all BC-ECG signals.

Finally it is noteworthy that the normalized amplitudes in BC-ECG vary for the different rings of the multi-ring electrode. The smaller rings emphasize the local activity in the electrode surroundings, yielding higher NA values for the dipole sources nearest to the electrode. Conversely, the active cardiac dipoles from distant regions are greatly attenuated, especially in the case of the smallest rings, resulting in the poor D values of cardiac waves associated with distant sources. This means that both the detectability (D) and the normalized amplitude (NA) of the cardiac waves depend on the relative position of the electrodes, the 

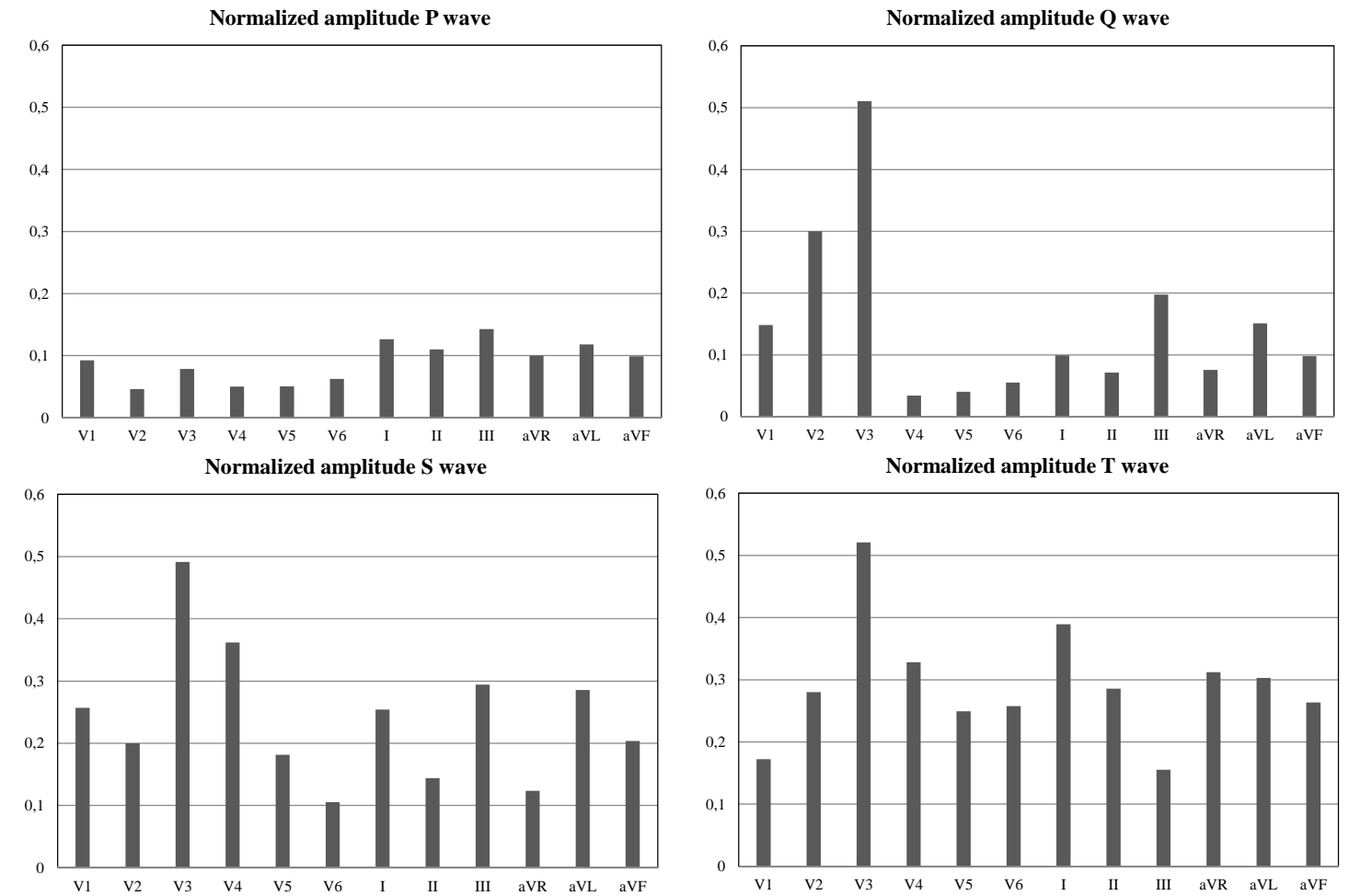

Figure 5. Bar charts of normalized amplitudes of the P, Q, S and T waves for the standard 12-Lead ECG recordings. 

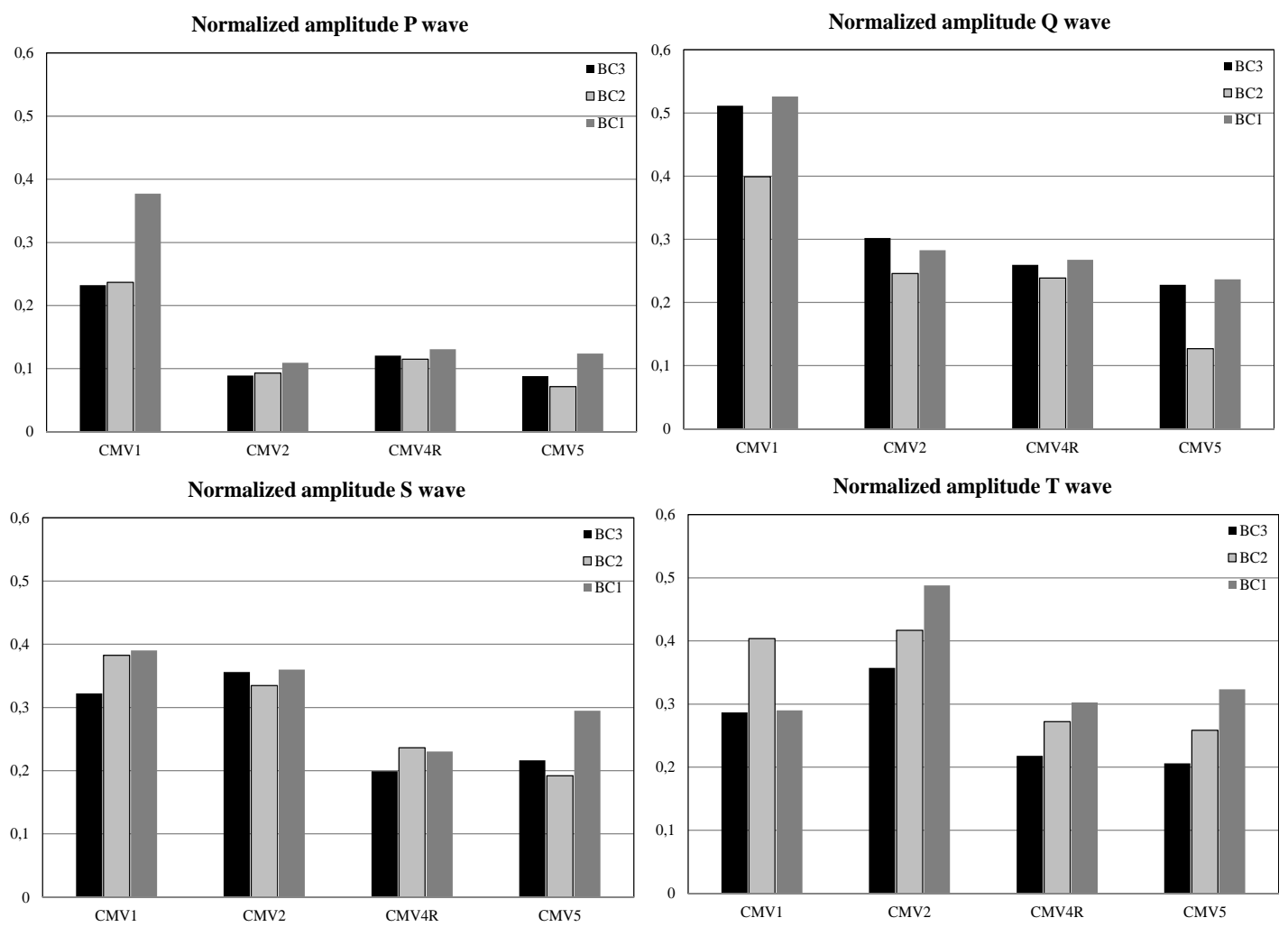

Figure 6. Bar charts of normalized amplitudes corresponding to $\mathrm{P}, \mathrm{Q}, \mathrm{S}$ and $\mathrm{T}$ waves for the standard multi-ring electrode locations.

DxNA P wave (\%)

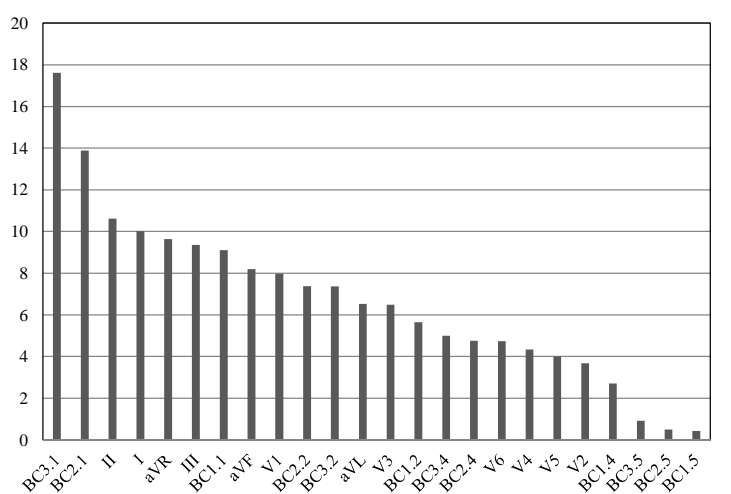

DxNA S wave (\%)

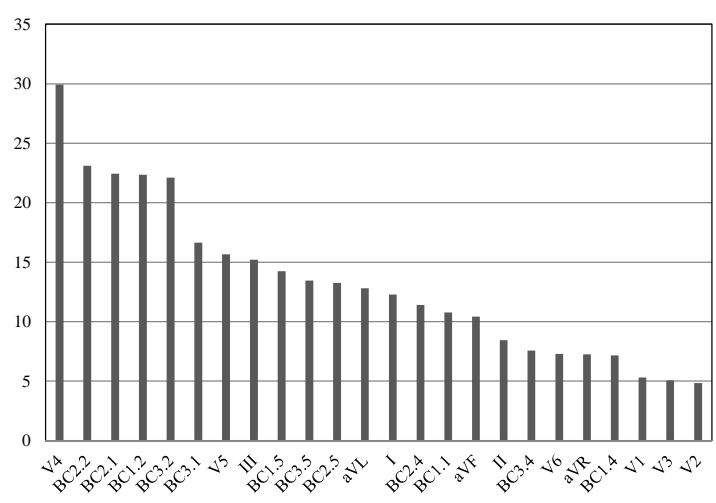

DxNA Q wave (\%)

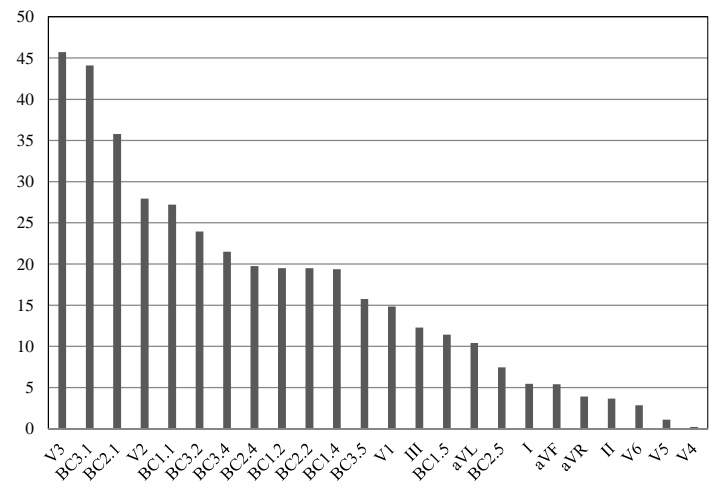

DxNA T wave $(\%)$

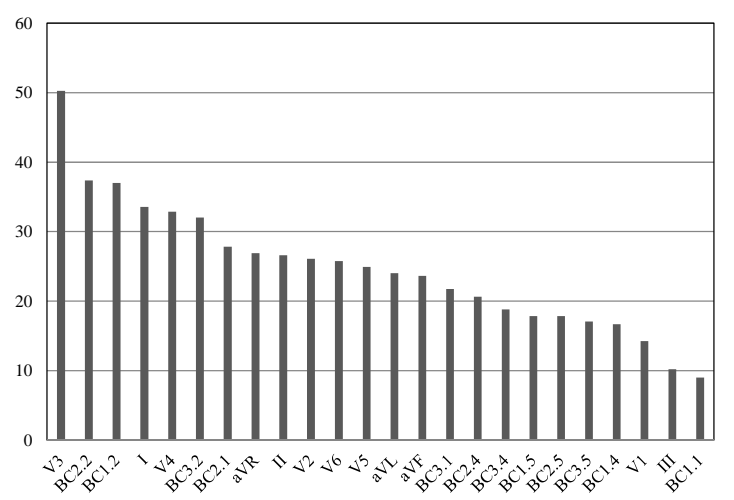

Figure 7. Bar charts of DxNA values in descending order of the P, Q, S and T waves for all ECG recordings (standard \& multi-ring). 

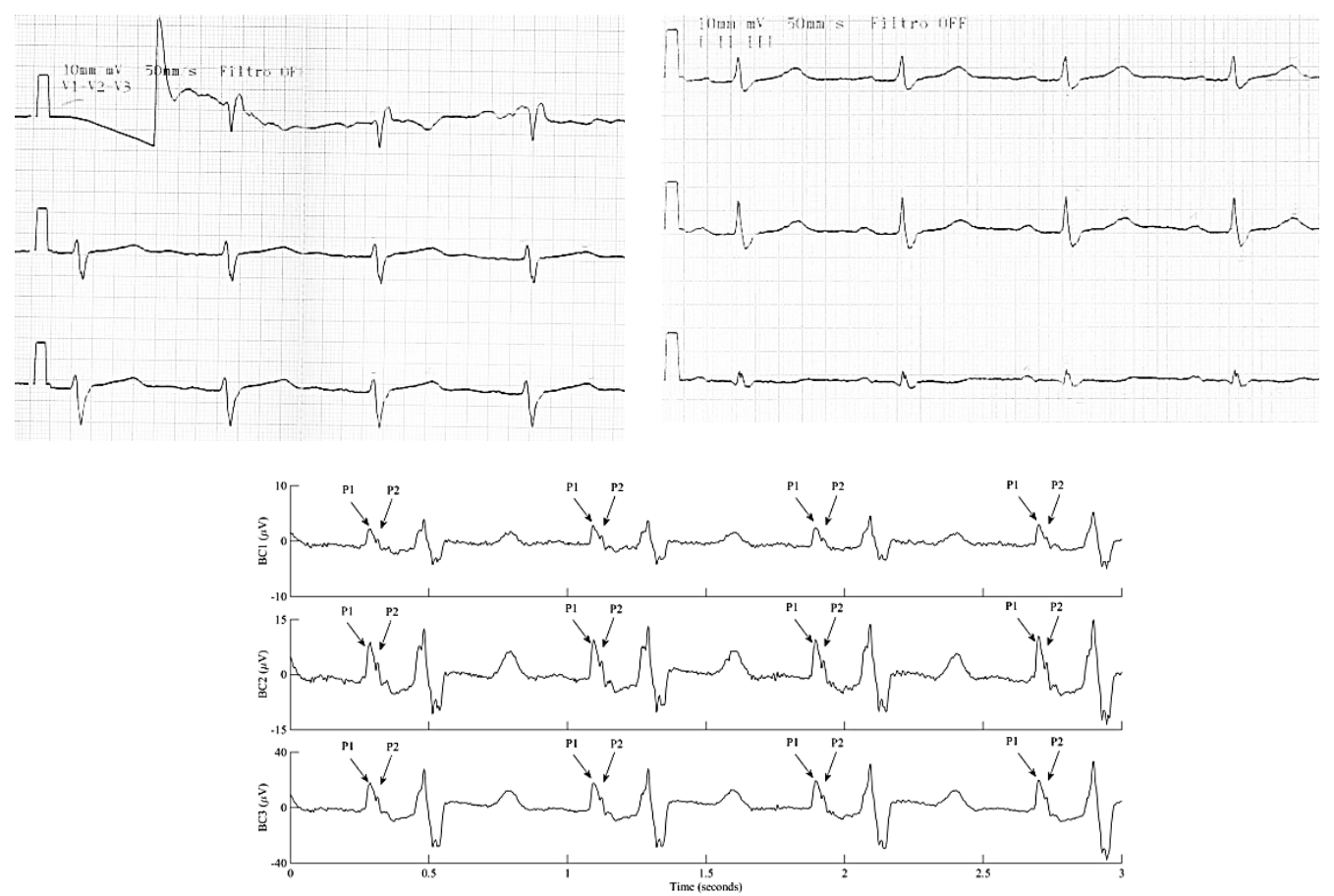

Figure 8. Top: V1, V2 and V3 (left) and I, II and III (right) ECG leads and; Bottom: BC-ECG signals obtained at CMV1 in the same subject. P1 and P2 waves of atrial activity can be clearly identified in all BC-ECG traces but not in the standard ones. Furthermore this patient's right bundle branch block, although visible in Leads V2 and V3 is highly detailed in BC-ECG signals. 

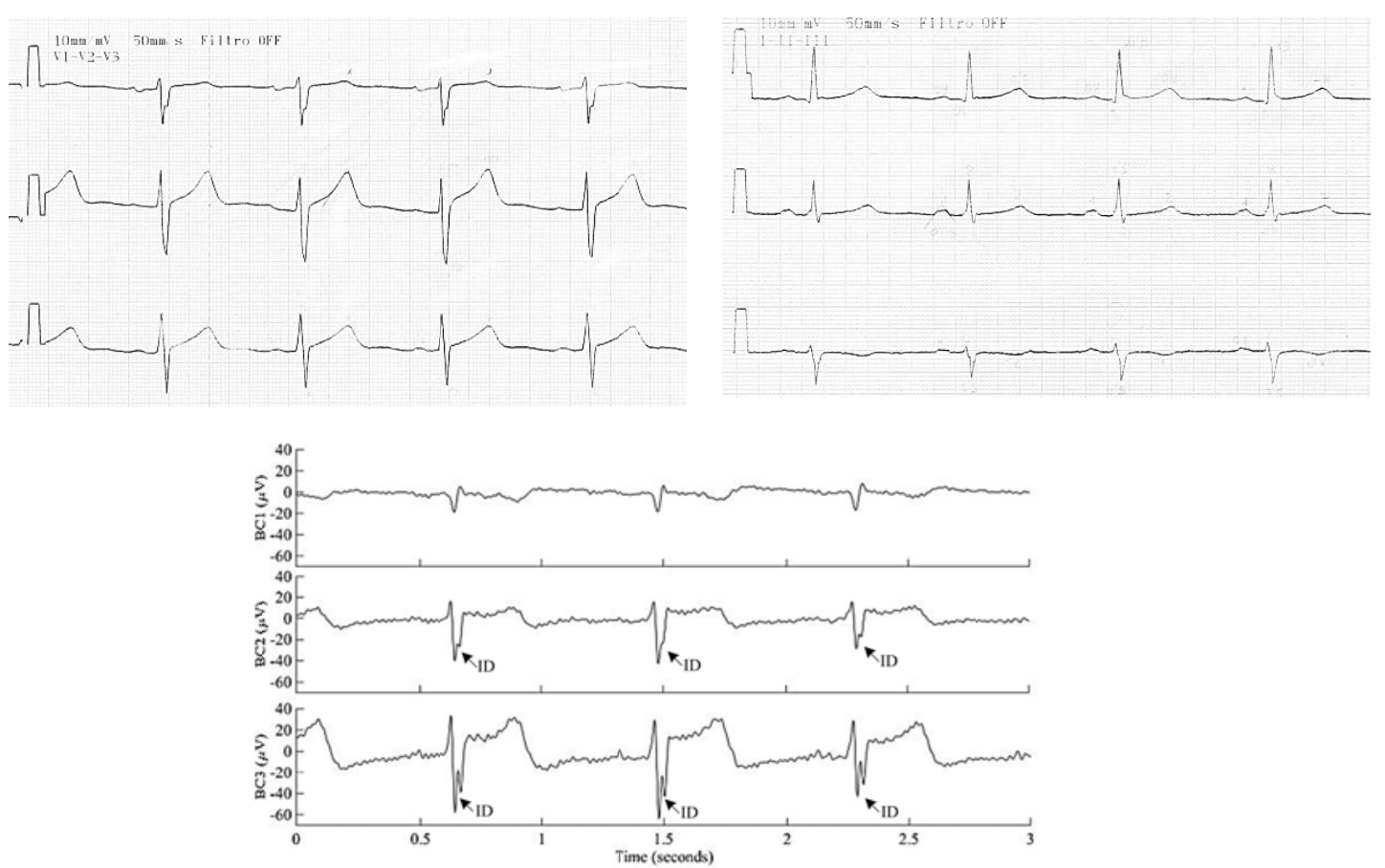

Figure 9. Top: V1, V2 and V3 (left) and I, II and III (right) ECG leads; Bottom BC-ECG signals obtained in CMV2 position for the same subject. Although impaired intraventricular driving (ID) can be observed in the standard ECG, this is more evident in BC-ECG signals.
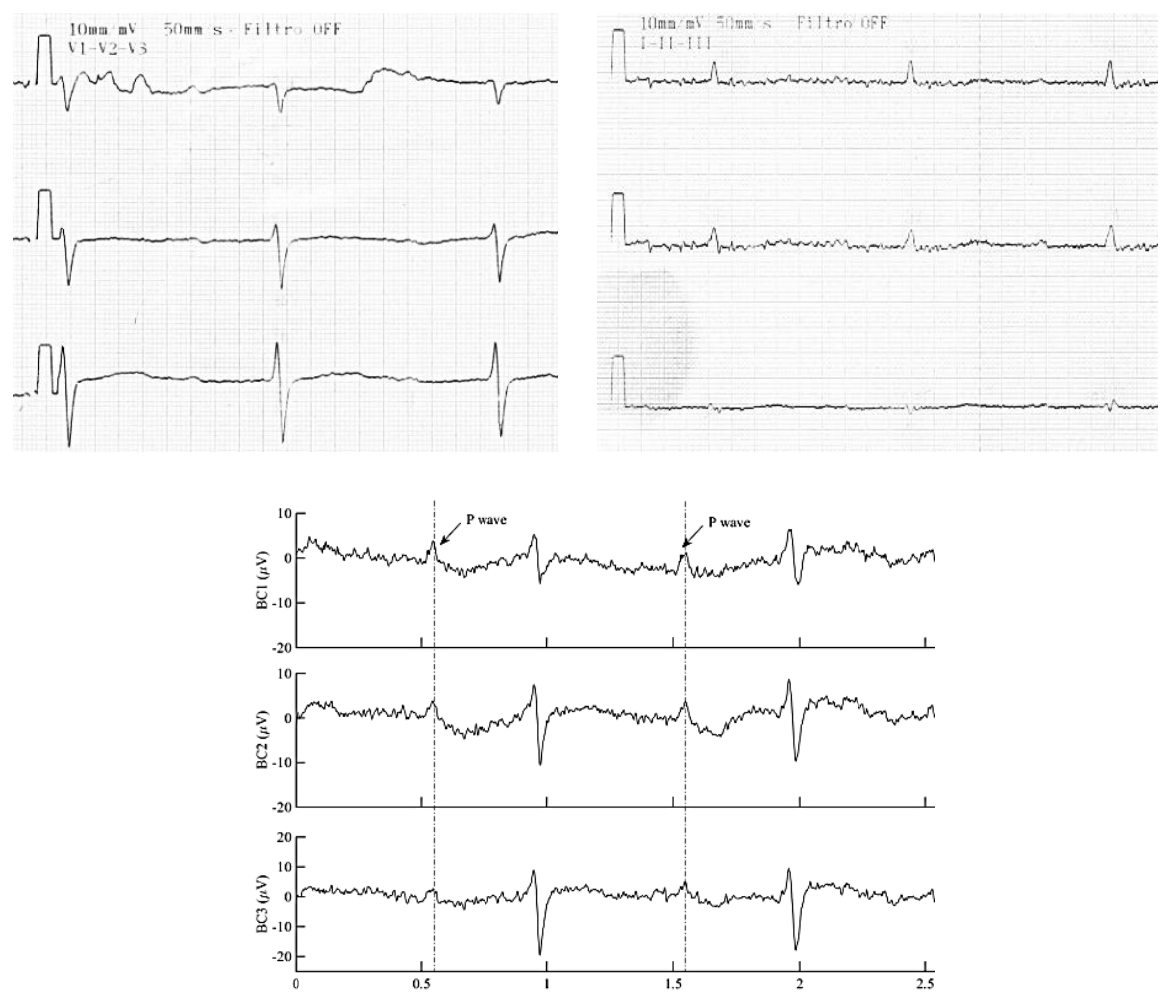

Figure 10. Top V1, V2 and V3 (left) and I, II and III (right) ECG leads; Bottom BC-ECG signals obtained at CMV1 for the same subject. Patient with atrioventricular block. Atrial activity is more evident in BC-ECG recordings. 


\section{Discussion}

Although several studies have shown that BC-ECG recordings have higher spatial resolution than those obtained from monopolar electrodes [4-6] and tripolar concentric wired electrodes have been used for cardiac mapping and compared to standard Lead II-ECG signals [6], as far as we know the present study is the first to compare the standard 12-lead system with electrocardiographic recordings by four multi-CREs. It is also the first related to BC-ECG recordings by a multi-CRE with wireless transmission and / or data storage in $\mu \mathrm{SD}$.

Our results show that the 12-lead standard system is better than the BC-ECG system proposed here in detecting the cardiac waves (PQRST). However, this superiority is compromised if fewer ECG-leads are used, as in the case of many ECG holters. In this regard, detectability values in BC-ECG signals are better for nodes at CMV1 and CMV2 than at CMV4R and CMV5 for all electrocardiographic waves. If slight changes are made to the electrode positions, instead of setting a 'blind' fixed position, it is believed that higher detectability values could be achieved. Although it might have been useful to place a multi-CRE as near as possible to each precordial standard derivation, this procedure was discarded because the electrodes were too big for this purpose, as our intention was to analyze the effect of the multi-ring electrode size on BC-ECG signal recordings. This aspect will be considered in future studies.

In fact, the ring size is expected to affect the spatial sensitivity to pick up bioelectrical dipole sources [22]. In the present work it was seen that the larger the ring size the higher the BC-ECG amplitude picked up, which agrees with the findings of previous studies [14-22]. In this regard, BC-ECG amplitudes ranged from a few microvolts to a hundred microvolts, which is within the range of values reported in the literature [6-15]. In addition, for the same multi-ring electrode, the ability to detect the different ECG waves is lower for BC1 (21.5 mm outer diameter ring) than for BC2 (33.5 mm) and BC3 (45.5 mm) ECG recordings. Therefore, the distance between the ring and central disk should be greater than $21.5 \mathrm{~mm}$ (BC1 outer diameter) so as to better capture the underlying electrocardiographic activity. It is noteworthy that increasing the ring size from $21.5 \mathrm{~mm}$ to $33.5 \mathrm{~mm}$ (BC2 outer diameter) yields significantly higher cardiac wave detectability rates, but the improvement is less significant when changed from $33.5 \mathrm{~mm}$ to $45.5 \mathrm{~mm}$ (BC3 outer diameter). This suggests that a $33.5 \mathrm{~mm}$ external diameter CRE picks up signals with a good compromise between the enhanced usability and spatial resolution of smaller electrodes and the better detectability and higher amplitudes of signals from larger ring electrodes. On the other hand, in order to be able to study cardiac waves for the diagnosis of cardiac disorders, these waves should not only be detectable but they should also have a signicant relative amplitude in the recorded signal. In this sense, a new parameter (DxNA) was defined to estimate the ability of the different electrode configurations to pick up different electrocardiographic waves.

This parameter has shown the advantage of the BC-ECG signals (BC3 and BC2 recordings at CMV1) in picking up atrial activity ( $\mathrm{P}$ wave) over standard 12-Lead recordings. This is especially relevant considering that $\mathrm{P}$ wave amplitude is the lowest of the electrocardiographic waves, due to the number of cells involved in atrial activity being much lower than those involved in ventricular activity. The results obtained by BC3.1 and BC2.1 in picking up the $\mathrm{P}$ wave (DxNA=17.8 and 13.9) are far superior to the best values associated with standard leads (I, II; 10.5 and 9.8, respectively). This is of particular interest, since these leads could not be monitored by holter devices. If only precordional leads are considered, the superiority of BC-ECG signals is even more evident (V1: 9). For Q, S and T waves, the highest 
values are reached in the V3 or V4 precordial leads, but closely followed by BC3 and BC2 recordings at CMV1 or CMV2. Higher values for DxNA could have been reached for the BCECG recordings if the multi-CREs had been placed at positions comparable to V3 and V4. Also, as regards the location of the multiring electrode nodes, it seems that CMV4R and CMV5, which presented lower DxNA values than CMV2 and CMV1, do not provide significant additional information and therefore may not be considered in further studies.

Apart from DxNA parameter values, it has been shown that BC-ECG recordings provide better spatial resolution in picking up cardiac activity. Indeed, in most of the BC-ECG signals picked up at CMV1, P1 and P2 atrial waves were clearly identified [1]. Likewise, ECG signals obtained from volunteers with different pathologies associated with local regions with anomalous electrical activity (such as impaired intraventricular driving, or AV block) reveal the higher spatial resolution of BC-ECG signals compared to standard monopolar 12-ECG recordings. It is worthy of note that this enhancement is achieved by means of the electrode configuration (ring electrodes) without the need for invasive procedures such as catheterization.

Regarding technical aspects, the multi-CRE sensor node used in this study represents an advance on a previous prototype developed by the present research group that allowed simultaneous recording, conditioning and wireless transmission of one BC-ECG signal captured by a tripolar concentric disposable and flexible sensing electrode and Lead-I ECG picked up using monopolar conventional electrodes [16]. This module was inadequate to meet the requirements of the current application, in which three bipolar bioelectric signals are transmitted by each multi-CRE sensor node with higher sampling frequency and better amplitude resolution. We should emphasize that despite the fact that the amplitudes of the 12-Lead ECG $(\mathrm{mV})$ are much higher than those of BC-ECG signals (tens-hundreds of $\mu \mathrm{V}$ ) it is observed that the present conditioning, storage and $\mathrm{BC}$ wireless transmission system provides high quality signals, comparable to those obtained with a standard 12-lead ECG using commercial systems with SNR ratios higher than 35dB similar to those obtained in [16].

The next logical step in introducing CRE into clinical practice would be to compare standard 12-lead ECG and BC-ECG recordings obtained by placing CRE electrodes with an external diameter of $33.5 \mathrm{~mm}$ or more in the standard precordial positions. Nevertheless, these positions may not be the optimal for BC-ECG recordings. In view of the spatial selectivity of CRE electrodes, a matrix of these electrodes should be placed over the heart to provide enhanced recordings of atrial activity. This could provide improved signal analysis and cardiac isochronal activation maps so as to accurately identify the cardiac electrical activity and could be of help in the diagnosis of conditions such as fibrillation or atrial flutter, ventricular arrhythmias, atrial hypertrophy or ventricular ischemia [7].

Regarding hardware, we also plan to develop a System on Chip (SoC) integrated version of the multi-CRE sensor node hardware, which would make it possible to significantly reduce its dimensions and power consumption [23-25]. Likewise, we intend to expand the multi-CRE sensor node bandwidth to [0.05-150 Hz] so as to prevent loss of information of the ECG bandwidth at low frequencies, to improve the diagnosis of certain cardiac pathologies in which these components are particularly significant, as in the case of ischemias. Finally, the system presented in the present work was originally intended to increase the local spatial resolution achieved from standard 12-Lead ECG signals in short-term clinical recordings with patients at rest, offering additional patient comfort and ease of use. A comprehensive study aimed at obtaining ECG recordings in patients in motion and with minimal skin preparation should be undertaken to assess the applicability of the developed wireless system in ambulatory ECG monitoring. 


\section{Conclusions}

An analysis was carried out of the effect of the position (CMV1, CMV2, CMV4R and CMV5) and dimensions (BC1, BC2 and BC3: 21.5, 33.5, $45.5 \mathrm{~mm}$ outer ring diameter) of a wireless multi-CRE node on the cardiac signal recorded, using a 12-Lead standard ECG as comparison.

Firstly, the conditioning, storage and wireless transmission nodes provide high quality BC2-ECG and BC3-ECG signals, similar to those of standard 12-lead recordings. The experimental results show that the bigger the ring size, the higher the amplitude of the BC-ECG signals. A $33.5 \mathrm{~mm}$ ring diameter picks up signals with a good compromise between better usability and the spatial resolution of smaller electrodes and the better detectability and higher amplitudes of the signals obtained from larger ring electrodes.

Secondly, the 12-Lead standard ECG is better able to detect cardiac waves than the proposed BC-ECG multi-node system. The detectability of electrocardiographic waves in BCECG signals is better at positions CMV1 and CMV2 than those obtained at CMV4R and CMV5.

Thirdly, BC-ECG signals (BC3 and BC2 recordings at CMV1) proved to be superior in picking up atrial activity, providing the best combination of detectability and normalized amplitude of the $\mathrm{P}$ wave.

Finally BC-ECG recordings can provide added value with respect to the classical electrocardiography when high spatial resolution electrocardiographic recordings are required, with no apparent significant contributions in other applications.

\section{Acknowledgments}

Research supported in part by: a grant from the Conselleria d'Educació, Cultura i Esport, Generalitat Valenciana Conselleria (GV/2014/029), two grants from the Universitat Politècnica de Valencia (SP20120490 and INNOVA SP20120469) and by a VLC_Campus grant (Prematuro 2013-0507)

\section{References}

1. Besio W and Tarjan PP 2002 Atrial activation Pattern from surface laplacian electrocardiograms of humans International Journal of Bioelectromagnetism 4 95-96

2. Lian J, Li G, Cheng J, Avitall B, and He B 2002 Body surface Laplacian mapping of atrial depolarization in healthy human subjects Med.Biol.Eng Comput. 40 650-659

3. He B and Cohen RJ 15-12-1992 Body surface Laplacian mapping of cardiac electrical activity Am.J.Cardiol. 70 1617-1620

4. Lu CC and Tarjan PP 2002 An ultra-high common-mode rejection ratio (CMRR) AC instrumentation amplifier for laplacian electrocardiographic measurement Biomed.Instrum.Technol. 33 76-83

5. Li GL, Lian J, Salla P, Cheng J, Ramachandra I, Shah P, Avitall B, and He B 2003 Body surface Laplacian electrocardiogram of ventricular depolarization in normal human subjects Journal of Cardiovascular Electrophysiology 14 16-27 
6. Besio $\mathrm{W}$ and Chen $\mathrm{T} 2007$ Tripolar Laplacian electrocardiogram and moment of activation isochronal mapping Physiol Meas. 28 515-529

7. Wang Y, Cuculich PS, Zhang J, Desouza KA, Vijayakumar R, Chen J, Faddis MN, Lindsay BD, Smith TW, and Rudy Y 2011 Noninvasive Electroanatomic Mapping of Human Ventricular Arrhythmias with Electrocardiographic Imaging (ECGI) Science Translational Medicine 3

8. SippensGroenewegen A, Peeters HA, Jessurun ER, Linnenbank AC, Robles de Medina EO, Lesh MD, and van Hemel NM 3-2-1998 Body surface mapping during pacing at multiple sites in the human atrium: P-wave morphology of ectopic right atrial activation Circulation 97 369-380

9. Kornreich F, MacLeod RS, and Lux RL 2008 Supplemented standard 12-lead electrocardiogram for optimal diagnosis and reconstruction of significant body surface map patterns J.Electrocardiol. 41 251-256

10. Fereniec M, Stix G, Kania M, Mroczka T, and Maniewski R 2014 An analysis of the Uwave and its relation to the T-wave in body surface potential maps for healthy subjects and MI patients Ann.Noninvasive.Electrocardiol. 19 145-156

11. He B, Li GL, and Lian J 2002 A spline Laplacian ECG estimator in a realistic geometry volume conductor IEEE Transactions on Biomedical Engineering 49 110-117

12. Prats-Boluda G, Garcia-Casado J, Martinez-de-Juan JL, and Ye-Lin Y 2011 Active concentric ring electrode for non-invasive detection of intestinal myoelectric signals Med.Eng.Phys. 33 446-455

13. Koka K and Besio WG 30-9-2007 Improvement of spatial selectivity and decrease of mutual information of tri-polar concentric ring electrodes J.Neurosci.Methods 165 216222

14. Kaufer M, Rasquinha L, and Tarjan P 1990 Optimization of multi-ring sensing electrode set 12 612-613

15. Prats-Boluda G, Ye-Lin Y, Garcia-Breijo E, Ibanez J, and Garcia-Casado J 2012 Active flexible concentric ring electrode for non-invasive surface bioelectrical recordings Measurement Science \& Technology 23

16. Prats-Boluda G, Ye-Lin Y, Barrachina JMB, Senent E, de Sanabria RR, and GarciaCasado J 2015 Development of a portable wireless system for bipolar concentric ECG recording Measurement Science \& Technology 26

17. Gussak I, Wright RS, Bjerregaard P, Chaitman BR, Zhou SH, Hammill SC, and Kopecky SL 2000 False-negative and false-positive ECG diagnoses of Q wave myocardial infarction in the presence of right bundle-branch block Cardiology 94 165-172

18. Shin, H., Lee, C., and Lee, M. 2007 Principal Point Discrimination of Electrocardiogram for Automatic Diagnosis IFMBE Proceedings 14 1087-1090

19. Javadi M 2014 On the post-design aspects of human/animal electrocardiogram P-QRS-T detection algorithms Scientia Iranica 21 425-437 
20. Sovilj S, Van OA, Rajsman G, and Magjarevic R 2010 ECG-based prediction of atrial fibrillation development following coronary artery bypass grafting Physiol.Meas. 31 663677

21. Fratini A, Sansone M, Bifulco P, and Cesarelli M 2015 Individual identification via electrocardiogram analysis Biomed.Eng.Online. 14 78-

22. Farina D and Cescon C 2001 Concentric-ring electrode systems for noninvasive detection of single motor unit activity IEEE Trans.Biomed.Eng 48 1326-1334

23. Khayatzadeh M, Zhang X, Tan J, Liew WS, and Lian Y 2013 A 0.7-V 17.4- mu W 3-lead wireless ECG SoC IEEE Trans.Biomed.Circuits Syst. 7 583-592

24. Morrison T, Nagaraju M, Winslow B, Bernard A, and Otis BP 2014 A $0.5 \mathrm{~cm}(3)$ FourChannel $1.1 \mathrm{~mW}$ Wireless Biosignal Interface With $20 \mathrm{~m}$ Range IEEE Transactions on Biomedical Circuits and Systems 8 138-147

25. Tan J, Liew WS, Heng CH, and Lian Y 2014 A 2.4 GHz ULP Reconfigurable Asymmetric Transceiver for Single-Chip Wireless Neural Recording IC IEEE Transactions on Biomedical Circuits and Systems 8 497-509 